\title{
The Effect of Silver Diamine Fluoride in Preventing Caries in the Primary Dentition: A Systematic Review and Meta-Analysis
}

\author{
Branca Heloisa Oliveira ${ }^{a}$ Anjana Rajendra ${ }^{b}$ Analia Veitz-Keenan ${ }^{c}$ \\ Richard Niederman ${ }^{b}$
}

${ }^{a}$ Department of Community and Preventive Dentistry, Faculty of Dentistry, University of the State of Rio de Janeiro, Rio de Janeiro, Brazil; ${ }^{b}$ Department of Epidemiology and Health Promotion, College of Dentistry, New York University, New York, NY, USA; ' Department of Oral and Maxillofacial Pathology, Radiology and Medicine, College of Dentistry, New York University, New York, NY, USA

\section{Keywords}

Cariostatic agents - Dental caries · Preventive dentistry ·

Silver diamine fluoride $\cdot$ Systematic review

\begin{abstract}
Objectives: To investigate whether silver diamine fluoride (SDF) is effective in preventing new caries lesions in primary teeth when compared to placebo or active treatments. Methods: Systematic review (CRD42016036963) of controlled clinical trials. Searches were performed in 9 electronic databases, 5 registers of ongoing trials, and reference lists of identified review articles. Two researchers carried out data extraction and quality appraisal independently. The primary outcome was the difference in caries increment (decayed, missing, and filled surfaces or teeth - dmfs or dmft) between SDF and control groups. These differences were pooled as weighted mean differences (WMD) and prevented fractions (PF). Results: Searches yielded 2,366 unique records; 6 reports of 4 trials that randomized 1,118 and analyzed 915 participants were included. Two trials compared SDF to no treatment, 1 compared SDF to placebo and sodium fluoride varnish (FV), and 1 compared SDF to high-viscosity glass ionomer cement $(\mathrm{GIC})$. All studies had at least 1 domain with unclear or high risk of bias. After 24 months of follow-up, in
\end{abstract}

comparison to placebo, no treatment, and FV, SDF applications significantly reduced the development of new dentin caries lesions (placebo or no treatment: WMD $=-1.15, \mathrm{PF}=$ $77.5 \%$; FV: WMD $=-0.43, \mathrm{PF}=54.0 \%$ ). GIC was more effective than SDF after 12 months of follow-up but the difference between them was not statistically significant (WMD, dmft: 0.34, PF:-6.09\%). Conclusion: When applied to caries lesions in primary teeth, SDF compared to no treatment, placebo or FV appears to effectively prevent dental caries in the entire dentition. However, trials specifically designed to assess this outcome are needed.

(c) 2018 S. Karger AG, Basel

Untreated dental caries of primary teeth was the tenth most common of 291 health problems assessed in the Global Burden of Disease 2010 Study [Marcenes et al., 2013]. Dental fear, financial limitations, scheduling difficulties and transportation issues are among the significant barriers to children's dental care reported by parents [Meyer et al., 2017]. These observations, when coupled with the fact that current methods of early preventive care do not appear to inhibit caries development [Blackburn et al., 2017] suggest the need for new or alternate approaches to control dental caries in children.

\section{KARGER}

(c) 2018 S. Karger AG, Basel

E-Mail karger@karger.com

www.karger.com/cre
Branca Heloisa Oliveira

Department of Community and Preventive Dentistry, Faculty of Dentistry University of the State of Rio de Janeiro

Boulevard 28 de Setembro, 157, Vila Isabel, Rio de Janeiro 20551-030 (Brazil)

E-Mail branca@uerj.br or branca.uerj@gmail.com 
Silver diamine fluoride (SDF) derives from the conjunction of silver nitrate and fluoride. It reduces the growth of cariogenic bacteria, hampers degradation of collagen in dentin, inhibits demineralization and promotes remineralization of both enamel and dentin [Zhao et al., 2018]. SDF has been used for decades in Japan [Nishino et al., 1969], China [Lo et al., 2001], Brazil [Bijella et al., 1991], and Argentina [Mauro et al., 2004] at concentrations varying from 10 to 38\% [Mei et al., 2016] in order to promote dental caries arrest. In 2014, after being approved by the Food and Drug Administration for tooth desensitization, it began to be used off-label for caries control in the USA [Horst et al., 2016].

The application of SDF to dental surfaces is simple and inexpensive [Mei et al., 2016] and complies with the concept of minimally invasive dentistry [Ericson et al., 2003]. Thus, treating caries lesions with SDF seems especially suitable for younger, less cooperative and more socially vulnerable children [Crystal and Niederman, 2016].

Multiple recently published systematic reviews indicate that SDF application successfully arrests dental caries in children [Duangthip et al., 2015; Gao et al., 2016a, b; Chibinski et al., 2017], and the use of SDF has been shown to be $89 \%$ (from 49 to $138 \%$ ) more effective in arresting dental caries in primary teeth than other active treatments or placebo [Chibinski et al., 2017]. Not heretofore systematically examined is the effect of SDF treatment on the development of new dental caries. That is, the effect of SDF on the prevention of new lesions in untreated dental surfaces of children who have had carious lesions treated with SDF or the prevention of new lesions in children who have had SDF applied to all primary teeth, irrespective of their caries status.

This review aims to investigate primarily whether SDF is superior to placebo or no treatment in preventing the development of new caries lesions in primary teeth. Its secondary purpose is to examine the preventive effect of SDF in comparison to other active treatments.

\section{Material and Methods}

This systematic review was registered in PROSPERO (CRD42016036963) and follows PRISMA reporting guidelines [Liberati et al., 2009].

We included all parallel-group, randomized or quasi-randomized, clinical trials with a follow-up of at least 1 year meeting the following inclusion criteria:

- participants: children 0-12 years of age;

- intervention: topical SDF solution (any concentration or frequency) applied by any health care worker at any settings;

Preventing Caries in Primary Teeth with SDF
Table 1. Search strategy developed for the Medline search

Search \#1 ("silver diamine fluoride" [Supplementary Concept] OR "silver diamine fluoride" [All Fields]) OR ("silver fluoride" [Supplementary Concept] OR "silver fluoride" [All Fields]) OR ("silver nitrate" [MeSH Terms] OR ("silver" [All Fields] AND "nitrate" [All Fields]) OR "silver nitrate" [All Fields]) OR ("“silver" [MeSH Terms] OR "silver" [All Fields]) AND ("nanoparticles" [MeSH Terms] OR "nanoparticles" [All Fields] OR "nanoparticle" [All Fields]))

Search \#2 (((( (tooth demineralization[MeSH Terms] $)$ OR caries) OR dental decay) OR cavit*) OR tooth remineralization) OR tooth discoloration

\#1 AND \#2

- comparisons: no intervention, placebo, any topical cariostatic agents, resin or glass ionomer pit and fissure sealants or dental restorative materials;

- outcomes: primary outcome was the development of new dentin caries lesions at surface, tooth, or patient level. The secondary outcome measures were any self/parent-reported or professionally diagnosed adverse events.

Studies that did not meet one of these criteria (e.g., case series, guidelines, letters to the editor, trials where participants were older than 12 years of age) were excluded.

The searches were performed in April 2016 and updated in July 2017 , without language or date of publication restraints, in the Cochrane Central Register of Controlled Trials (CENTRAL), Embase, Medline via PubMed, Scopus, Web of Science, Lilacs, BBO and Scielo databases. Additionally, 5 registers of ongoing trials and 1 database of theses and dissertations were searched: ClinicalTrials.gov, Brazilian Register of Clinical Trials, EU Clinical Trials Register, ISRCTN registry and Current Controlled Trials, ANZCTR-Australian New Zealand Clinical Trials Register, and Capes dissertations database. A highly sensitive search strategy was initially developed for Medline and later adapted for the other databases and repositories of trials with the help of a librarian. Being aware that SDF is primarily used for caries arrest, our search used terms that would allow us to identify trials where SDF was used for caries arrest. However, such trials would only be included in the review if they also provided data on caries prevention (Table 1). Cross-referencing from reviews on the subject of SDF for caries prevention or arrest was used to identify further potential articles.

The records were uploaded to one core database using EndNote X7 (Thomson Reuters, San Francisco, CA, USA), and duplicates were removed. Two reviewers (B.H.O. and A.R.) pilot tested the eligibility criteria on a sample of 17 reports including ones that were thought to be definitely eligible, definitely not eligible, and doubtful. After independently assessing these reports, they discussed the difficulties encountered in applying the eligibility criteria. During this phase only 1 disagreement occurred. Then, they independently examined the title and abstracts of each of the remaining reports. When a study apparently met our inclusion criteria but no abstract was available, the full article was obtained and read. Studies in languages other than English had the data extracted with the help of individuals knowledgeable in those languages. 


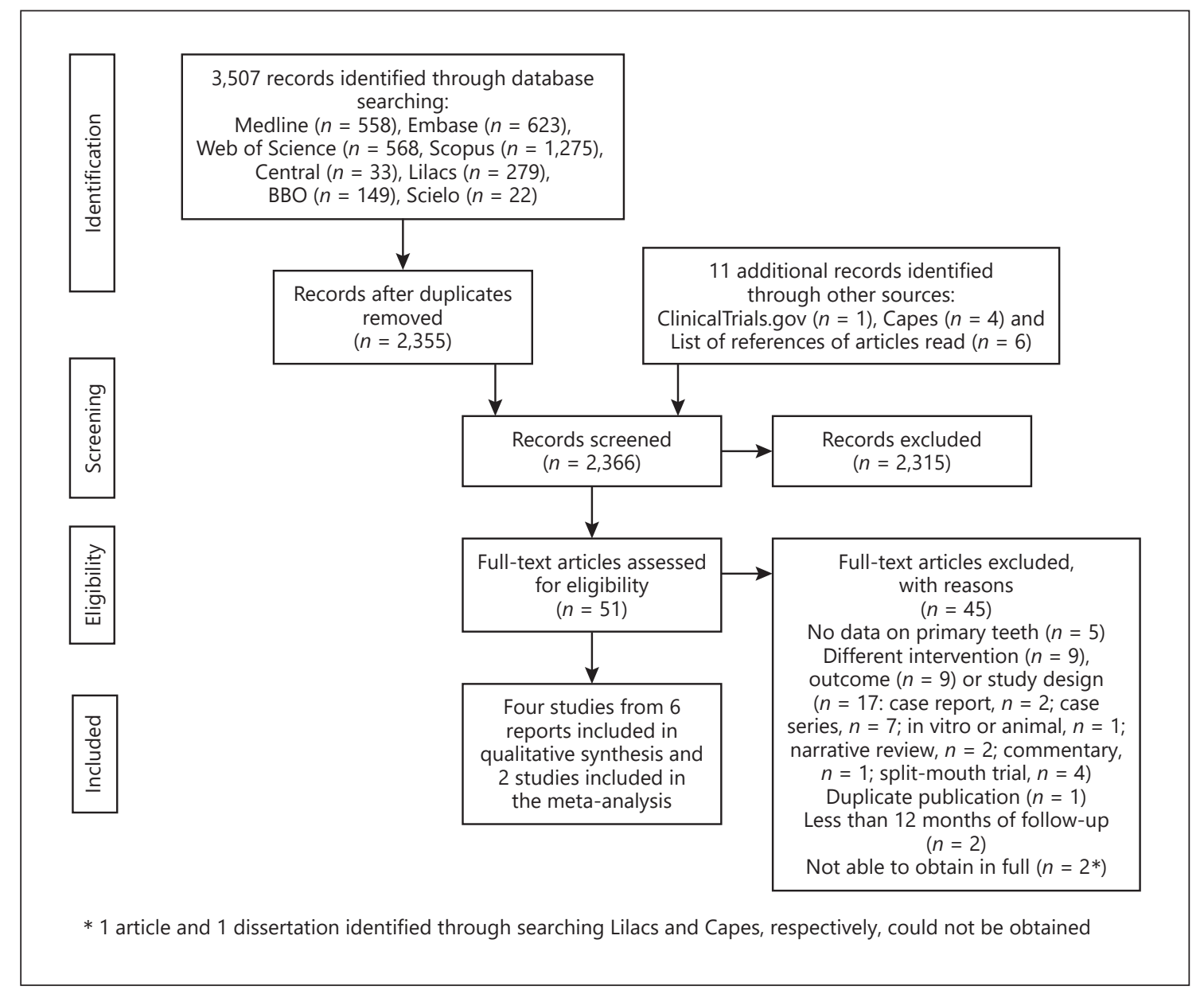

Fig. 1. Flow diagram showing the process of identifying, screening, assessing for eligibility, excluding, and including studies.

We attempted to contact study authors to obtain missing or unclear information.

Two review authors independently read all the studies selected for inclusion, extracted the data and assessed their risk of bias by using the Cochrane risk of bias tool [Higgins and Green, 2011]. Disagreements between the review authors over the risk of bias in particular studies was resolved by consensus with involvement of a third review author where necessary.

The primary measure of treatment effect was the difference in mean caries increment at dentin level between SDF and control groups (MD; mean new decayed, filled, and extracted tooth surfaces/teeth in the test group minus mean new decayed, filled, and extracted tooth surfaces/teeth in the control group). The prevented fraction (PF; mean increment in control minus mean increment in intervention groups divided by mean increment in control) was the secondary measure of treatment effect. When there were more than 1 relevant intervention and/or comparison groups they were combined into a single intervention and/or comparison group [Lo et al., 2001; Chu et al., 2002]. These procedures were performed as recommended in the Cochrane Handbook for Systematic Reviews of Interventions [Higgins and Green, 2011]. Confidence intervals of PF were calculated using Fieller's method [Abrams et al., 1972]. Since the estimate of the between-studies variance under the random-effects model has poor precision when the number of studies is very small [Borenstein et al., 2009], we used the fixed-effect model and the inverse variance method to obtain pooled estimates of caries increment as weighted mean differences (WMD) and PF when fewer than 4 studies were combined. Heterogeneity of studies was assessed by the $\chi^{2}$ test for heterogeneity and the Higgins index $\left(\mathrm{I}^{2}\right)$. The studies in our meta-analyses were grouped according to the duration of their follow-up in: less than 24 months and 24 months or more. Placebo or no intervention and active treatment (sodium fluoride varnish, FV, and glass ionomer cement, GIC) comparison groups were analyzed separately throughout. We only pooled study results when all necessary data could be obtained. All analyses were carried out in Stata ${ }^{\circledR} 14$ (StataCorp. LP, College Station, TX, USA). 
Table 2. Characteristics of the included studies

\begin{tabular}{|c|c|c|c|c|}
\hline Llodra, Cuba & 36 & $\begin{array}{l}6 \text { or more (mean } 6.29 \text {, } \\
\text { SD } 0.48) \\
\text { dmfs }=3.35-3.68\end{array}$ & $\begin{array}{l}38 \% \text { SDF applications for } 3 \mathrm{~min} \text {, } \\
\text { onto all carious primary tooth } \\
\text { surfaces (every } 6 \text { months) }\end{array}$ & No treatment \\
\hline
\end{tabular}

SD, standard deviation; SDF, silver diamine fluoride; FV, 5\% sodium fluoride varnish; GIC, high-viscosity glass ionomer cement; $\mathrm{dfs}$, mean number of decayed and filled tooth surfaces in primary teeth; dft, mean number of decayed and filled primary teeth; dmfs mean number of decayed, missing, and filled tooth surfaces in anterior primary teeth; dmfs, mean number of decayed, missing, and filled tooth surfaces in primary teeth.

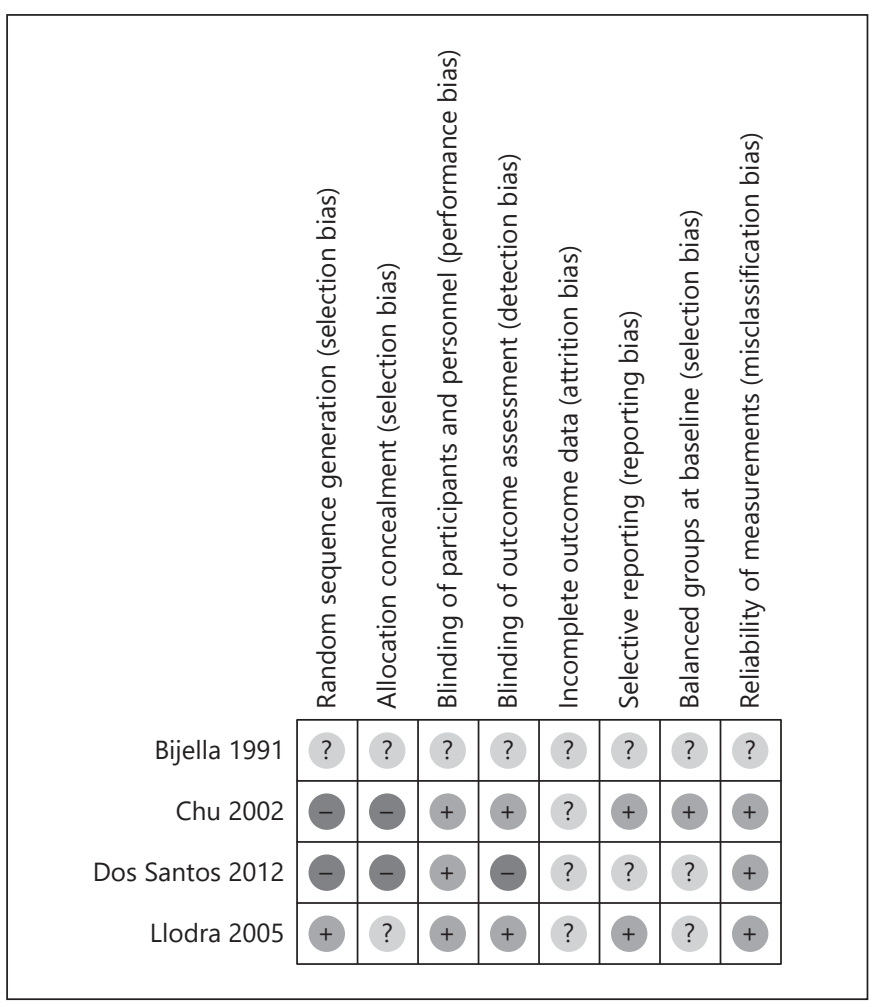

Fig. 2. Ascertainment of the risk of bias in the included studies. +, low risk; -, high risk; ?, unclear risk.

Preventing Caries in Primary Teeth with SDF

\section{Results}

The searches yielded 2,366 unique records. During the screening of titles and abstracts for identifying potentially eligible studies, 13 disagreements occurred and 10 of them resulted from simple oversights on the part of one of the reviewers and were solved by consensus after a brief discussion (e.g., a case report, 2 in vitro studies, a trial that tested the antimicrobial effects of a mouthwash containing silver nanoparticles). In the other 3 cases, a third researcher (R.N. or A.V.-K.) was consulted, and decision regarding the inclusion of the studies was reached by consensus. Fifty-one publications were read in full and assessed for eligibility. Six reports of 4 trials that randomized 1,118 and analyzed 915 participants were included (Fig. 1). At baseline, most participants had high caries experience, were not exposed to fluoridated water but were regularly exposed to some sort of topical fluoride product (i.e., fluoride toothpaste or $0.2 \%$ sodium fluoride school-based mouth rinse program). Two trials compared SDF to no treatment [Bijella et al., 1991; Llodra et al., 2005], 1 compared SDF to both a water placebo and 5\% sodium FV [Lo et al., 2001; Chu et al., 2002], and 1 compared SDF to high-viscosity GIC [Dos Santos, 2011; Dos Santos et al., 2012]. All trials, except for one [Bijella 


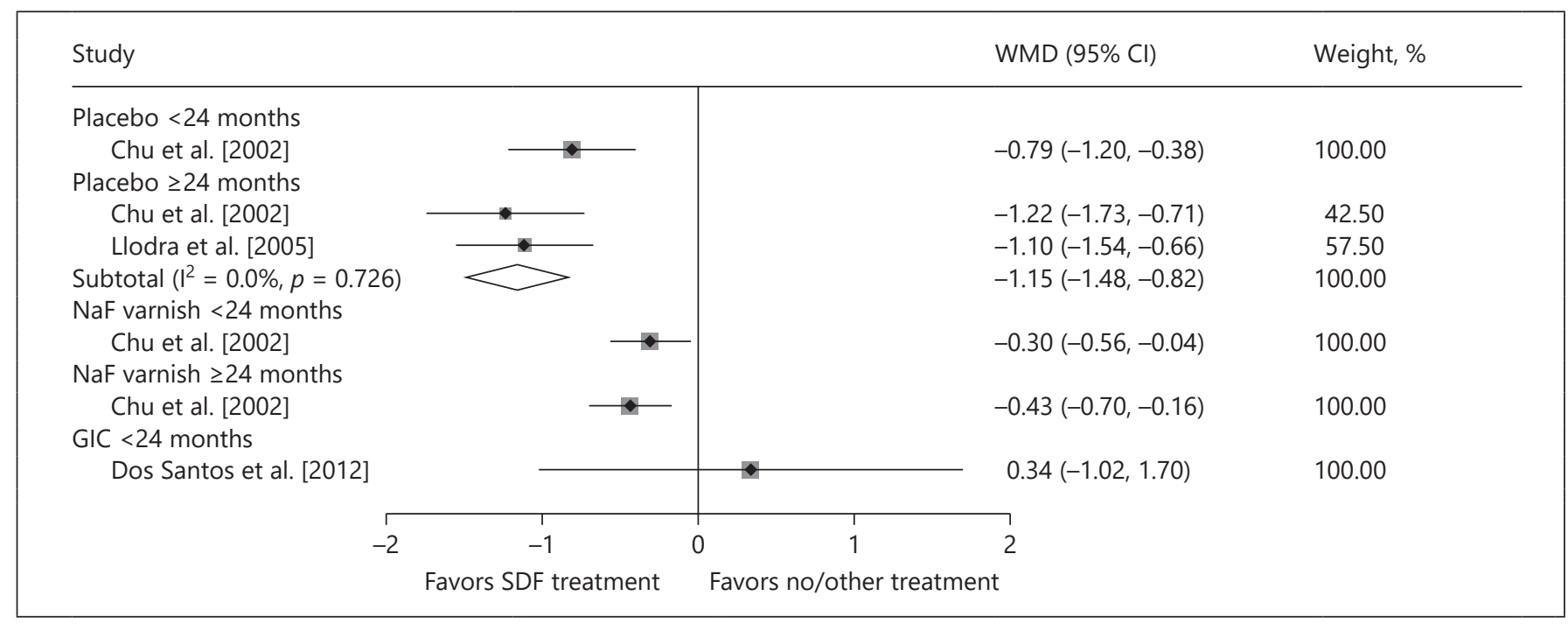

Fig. 3. Comparison between silver diamine fluoride and placebo (or no treatment), $5 \%$ sodium fluoride varnish (NaF varnish) and high-viscosity glass ionomer cement (GIC) regarding the mean increment in the number of decayed, extracted, and filled surfaces or teeth $(\mathrm{dmfs} / \mathrm{dmft})$ in the primary dentition, by duration of follow-up (24 months or more and less than 24 months). Only data on the comparison between SDF and GIC correspond to $\mathrm{dmft}$.

et al., 1991], applied SDF only to caries lesions with the aim of promoting caries arrest. The included trials differed regarding type of tooth surfaces treated, interval between SDF applications, and other aspects that are detailed presented in Table 2 .

All studies had at least 1 domain with unclear or high risk of bias (Fig. 2). One study [Bijella et al., 1991] was poorly reported and had insufficient information to permit judgment on high or low risk of bias in all domains. In 2 studies [Chu et al., 2002; Dos Santos et al., 2012] the investigators described the use of a non-random sequence generation process and in one study [Bijella et al., 1991] randomization was not mentioned. Blinding of participants and reliability of measurements by outcome assessors were classified as being at low risk of bias in all studies, except for 1 [Bijella et al., 1991]. Overall loss to followup was 15.4\% (12 months) [Dos Santos, 2011; Dos Santos et al., 2012], 21.5\% (24 months) [Bijella et al., 1991], 17.9\% (30 months) [Chu et al., 2002] and 17.5\% (36 months) [Llodra et al., 2005]; in the 4 trials, losses were similarly distributed in test and control groups. Bijella et al. [1991] attributed losses to children moving from school or city; Dos Santos [2011; Dos Santos et al., 2012] did not address missing outcome data; Llodra et al. [2005] and Chu et al. [2002] stated that baseline caries experience was similar in the analyzed test and control children but they did not clarify whether reasons for missing outcome data were balanced across groups.

Bijella et al. [1991] did not report standard deviations, and we were not able to contact any of the study authors. Therefore, this study was not included in the meta-analysis. Bijella et al. [1991] showed, after 24 months of follow-up, a 10,38, and 69\% decrease in caries incidence in primary tooth surfaces in the test groups (12\% SDF applications yearly, biannually and quarterly, respectively) in comparison to the control group (no treatment). Only the differences between quarterly versus yearly $12 \%$ SDF applications and quarterly $12 \%$ SDF applications versus no treatment were statistically significant.

The result of the meta-analysis of 2 studies [Chu et al., 2002; Llodra et al., 2005] comparing SDF applied to caries lesions to placebo or no treatment, with 24 months of follow-up or more, showed that SDF applications significantly reduced the development of dentin caries lesions in treated and untreated primary teeth (WMD: -1.15 ; 95\% CI: -1.48 to -0.82 and PF: $77.5 \%$; $95 \%$ CI: $67.8-$ 87.2\%). The comparisons between SDF and FV [Chu et al., 2002] and GIC [Dos Santos et al., 2012] were based on 1 study each; SDF performed significantly better than FV at 18 and 30 months of follow-up, and GIC performed better than SDF at 12 months of follow-up, although this difference was not statistically significant (Fig. 3, 4).
Oliveira/Rajendra/Veitz-Keenan/ Niederman 


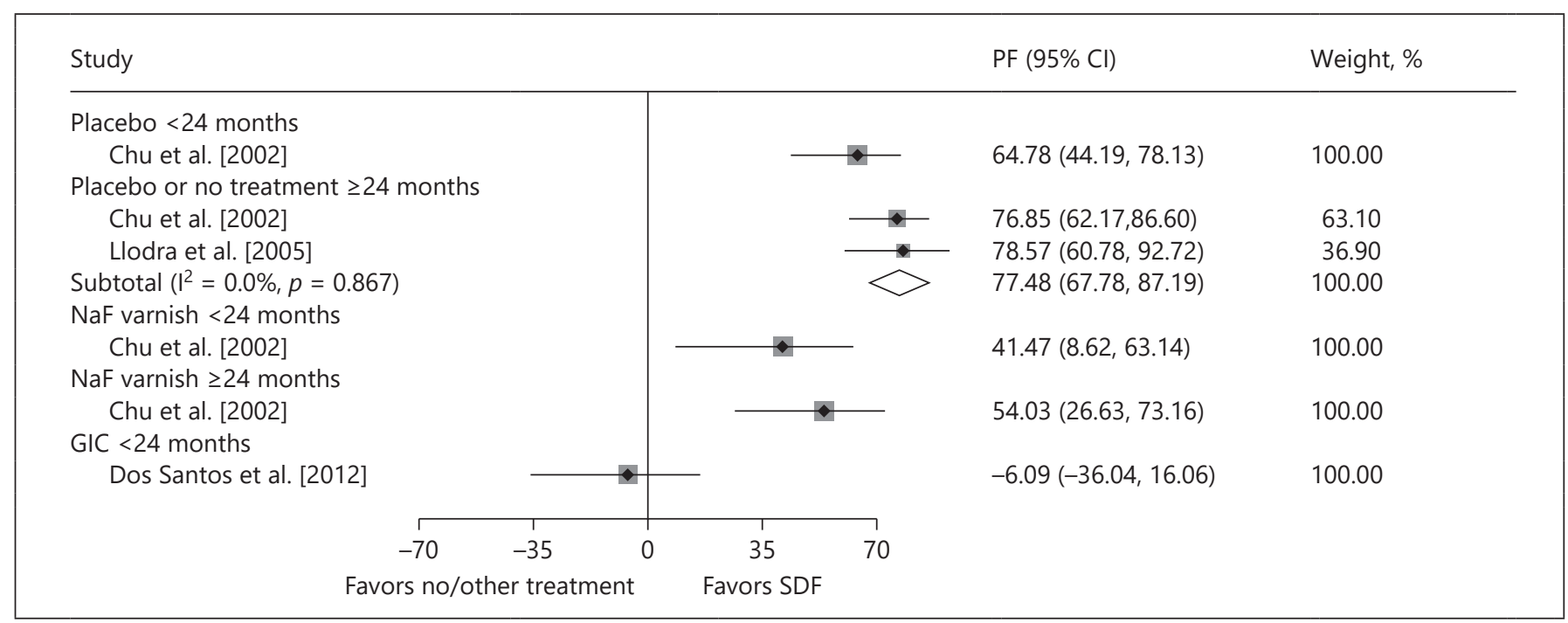

Fig. 4. Comparison between silver diamine fluoride and placebo (or no treatment), $5 \%$ sodium fluoride varnish ( $\mathrm{NaF}$ varnish) and high-viscosity glass ionomer cement (GIC) regarding the prevented fraction in the primary dentition, by duration of follow-up ( 24 months or more and less than 24 months).

We also performed a subgroup analysis combining the FV and placebo groups in the 2002 study of Chu et al. in order to estimate the size of SDF caries preventive effects in comparison to sodium FV applications or placebo. The WMD were -0.46 ( 1 study; $95 \%$ CI: -0.70 to -0.22 ) and -0.80 ( 2 studies; $95 \%$ CI -1.03 to -0.57 ) at less than 24 months of follow-up and at 24 months of follow-up or more, respectively. The PF were $49.3 \%$ (1 study; $95 \%$ CI: 29.9-68.6\%) and 71.2\% (2 studies; 95\% CI: 59.9-82.4\%) at less than 24 months of follow-up and at 24 months of follow-up or more, respectively.

Two studies mentioned that adverse events were assessed. In one, 3 patients "developed a small, mildly painful white lesion in the mucosa due to inadvertent contact with SDF solution that disappeared in $48 \mathrm{~h}$ without treatment" [Llodra et al., 2005]. In the other, it was reported that no side effects were observed during the 18-month follow-up period [Lo et al., 2001] and that parental satisfaction with children's dental appearance and dental health after 24 months of follow-up was similar between test and control groups [Chu et al., 2002]. Also, in these studies, the mean number of nonvital teeth at the end of the follow-up period was small and not significantly different between SDF and placebo or no treatment groups.

Preventing Caries in Primary Teeth with SDF

\section{Discussion}

This systematic review shows that when SDF is used to arrest caries lesions in primary teeth it also provides an anticaries benefit for the entire dentition; that is, $38 \%$ SDF applications decrease by $77 \%$ the development of new caries in treated children in comparison to nontreated children ( 2 studies; 558 participants). This estimate of effect, although large, is based on 2 studies [Chu et al., 2002; Llodra et al., 2005] whose potential limitations may lower our confidence in their findings [Balshem et al., 2011].

One study with unclear risk of bias in all domains that was not included in the meta-analysis [Bijella et al., 1991] investigated whether the frequency of $12 \%$ SDF applications on all tooth surfaces, irrespective of their caries status, would influence the SDF preventive effect. It showed that caries incidence after quarterly SDF applications was significantly lower than after yearly applications but not significantly different from caries incidence after biannual applications. Although it has also been demonstrated that for dental caries arrest biannual SDF applications are superior to yearly SDF applications [Fung et al., 2016], the ideal interval for SDF applications is still a matter of debate and deserves further investigation [American Academy of Pediatric Dentistry, 2017].

Regarding the preventive effect of SDF in comparison to other active treatments, only 1 study with sodium FV 
[Chu et al., 2002] and 1 study with GIC [Dos Santos et al., 2012] met our inclusion criteria. The same occurred in the most recently published systematic review on SDF for caries arrest in primary teeth [Chibinski et al., 2017]. Taken together these highlight the need for more well-designed clinical trials on the topic.

The head-to-head comparison between yearly $38 \%$ SDF (44.800 ppm fluoride) and quarterly sodium FV (22.600 ppm fluoride) applications resulted in a 54\% (from 27 to $73 \%$ ) reduction in new caries experience in favor of SDF. Also noteworthy, while we found a $77 \% \mathrm{PF}$ for the 2 trials comparing SDF to placebo or no treatment in primary teeth, the corresponding PF in the Cochrane review on $\mathrm{FV}$ for caries prevention (10 trials) was 37\% [Marinho et al., 2013]. Thus, it is probably not necessary to combine SDF and FV applications in the same patient [American Academy of Pediatric Dentistry, 2017]. Still, the anticaries effect of SDF in comparison to or in combination with $\mathrm{FV}$ merits additional investigation.

The exact mechanism by which SDF may prevent the development of new caries lesions is still unknown, and one should not assume that its apparent greater benefit in comparison to FV is solely a consequence of its higher fluoride concentration. Laboratory studies have demonstrated that an insoluble protective layer of silver chloride and silver phosphate is formed on tooth surfaces following SDF application, decreasing calcium and phosphorus loss from demineralized enamel and dentin; the actual role of this silver compound in vivo requires additional investigation [Zhao et al., 2018].

In the study comparing painting on SDF with sealing cavities using GIC the point estimate favors GIC over SDF after 12 months of follow-up [Dos Santos et al., 2012]. However, the differences were not statistically significant. Caries clinical trials usually last from 2 to 4 years; the time necessary for significant numbers of lesions to develop at the cavitation level [Chesters et al., 2004] and statistically nonsignificant differences in caries after 12 months may become significant at 24 months [Chesters et al., 2002]. Recent work suggests that SDF application is far simpler and less expensive than sealants [Niederman et al., 2017]. Thus, a larger comparative effectiveness study lasting at least 24 months is needed. Supporting this need, although in situ and in vitro studies have shown that fluoride released from GIC may slow down the progression of dental caries lesions in adjacent tooth surface, its clinical effectiveness for adjacent caries prevention awaits clinical trials [Cury et al., 2015; Tedesco et al., 2016].

Finally, we examined potential side effects. It is well known that a high proportion of caries lesions arrested by the use of SDF become black [Gao et al., 2016a], and there is concern within dentistry about it [Nelson et al., 2016]. Only 1 of the included trials, where $38 \%$ SDF was applied to the upper primary teeth of Chinese kindergarten children, examined whether blackening of the carious lesions was upsetting to the child's parents. In contrast to the dentist's concerns, only $7 \%$ of the parents mentioned the presence of darkened teeth as the cause of their dissatisfaction with the child's dental appearance, and there were no significant differences among SDF, FV and placebo groups concerning parental assessment of the child's dental esthetics [Lo et al., 2001; Chu et al., 2002]. The extent to which these findings can be extrapolated to children of different cultural backgrounds is unknown, and the influence of tooth discoloration on treatment acceptability merits further investigation. For instance, a Web-based survey using photographs of carious teeth treated with SDF conducted in the USA found that parents considered staining on posterior teeth significantly more acceptable than staining on anterior teeth. However, most parents preferred anterior teeth staining to advanced behavioral techniques such as sedation or general anesthesia [Crystal et al., 2017].

Other postulated, but currently unreported, adverse effects include acute or chronic toxicity symptoms, mucosal or gingival inflammation signs and allergic episodes [Horst et al., 2016] and discomforts associated with SDF treatment (e.g., metallic taste or burning sensation) [Mei et al., 2016]. We did not identify any relevant problems of this nature in the included studies. Nevertheless, only data on the occurrence of pulp necrosis were systematically assessed in some individual trials [Lo et al., 2001; Chu et al., 2002; Llodra et al., 2005]. To actively collect and describe patient-reported outcomes is of utmost importance in clinical trials, and it should be routinely done in future SDF studies. It is also important to point out that the mean free fluoride and silver concentrations of $38 \%$ SDF are expected to be 44,800 and 255,000 ppm, respectively. Thus, although no episode of acute reaction or chronic intoxication caused by SDF was mentioned, SDF is not recommended for use in children that are allergic to silver compounds, and caution should be taken in order to avoid the application of a large quantity, particularly in very young children [Horst et al., 2016; Mei et al., 2013].

Although we performed a comprehensive search, we found only a very small number of clinical trials having the development of new caries lesions as outcome measure. Interestingly, the trials identified for this review were primarily designed to investigate the SDF potential
Oliveira/Rajendra/Veitz-Keenan/ Niederman 
for dental caries arrest and most applied SDF to carious lesions only [Lo et al., 2001; Chu et al., 2002; Llodra et al., 2005; Dos Santos et al., 2012]. Among the 6 ongoing trials testing SDF in children that are currently registered at ClinicalTrials.gov, all were designed to examine the effect of SDF in caries lesions, and only 1 of them will assess caries incidence in other teeth. Thus, whether SDF applied to tooth surfaces (e.g., pits and fissures), irrespective of their caries status, would have an important role in dental caries prevention is yet to be investigated, and trials specifically designed to address this outcome should be encouraged.

Since SDF topical applications are simple, safe, inexpensive, and have the potential to both arrest and prevent dental caries in primary teeth, they may have a significant impact on reducing the burden of untreated dental disease in children. Nevertheless, the evidence on the preventive effect of SDF is based on only 2 small positive clinical trials with important limitations regarding study design and implementation. More rigorously designed studies, reported according to the Consolidated Standards of Reporting Trials Statement (CONSORT) [Schulz et al., 2010], are warranted to ensure unbiased high-quality evidence on the benefits of SDF applications for caries prevention.

\section{Acknowledgments}

We would like to thank Richard McGowan, MLS, Research Librarian at New York University College of Dentistry, for his help with searching the literature and Ms. Xiaoxi Gu and Dr. Mikako Deguchi, MBA, for their help with translating into English the ar- ticles written in Chinese and Japanese, respectively. We would also like to thank Dr. Ryan Richard Ruff for his valuable comments on some statistical aspects of the study and Dr. Dos Santos and Dr. Llodra who provided additional information about their trials. Research reported in this publication was partially supported by the National Institute on Minority Health and Health Disparities of the National Institutes of Health under Award No. R01MD011526 and U24MD006964, and partially funded through a Patient-Centered Outcomes Research Institute (PCORI) Award (PCS-160936824). The content is solely the responsibility of the authors and does not necessarily represent the official views of the National Institutes of Health or the Patient-Centered Outcomes Research Institute (PCORI), its Board of Governors, or Methodology Committee. The Teacher Training Program of the University of the State of Rio de Janeiro (Programa de Capacitação Docente da Universidade do Estado do Rio de Janeiro-PROCAD UERJ) also supported this work.

\section{Disclosure Statement}

The authors declare no potential conflicts of interest with respect to the authorship and/or publication of this paper.

\section{Author Contributions}

B.H. Oliveira contributed to conception, design, data acquisition, analysis, and interpretation, drafted and critically revised the manuscript; A. Rajendra contributed to conception, design, data acquisition, analysis, and interpretation, and critically revised the manuscript; A. Veitz-Keenan contributed to design, data analysis, and interpretation, and critically revised the manuscript; R. Niederman contributed to conception, design, data analysis, and interpretation, drafted and critically revised the manuscript. All authors gave final approval and agree to be accountable for all aspects of the work.

\section{References}

-Abrams AM, McClendon BJ, Horowitz HS: Confidence intervals for percentage reductions. J Dent Res 1972;51:492-497.

-American Academy of Pediatric Dentistry: Use of silver diamine fluoride for dental caries management in children and adolescents, including those with special health care needs. Pediatr Dent 2017;39:146-155.

Balshem H, Helfand M, Schunemann HJ, Oxman AD, Kunz R, Brozek J, Vist GE, Falck-Ytter Y, Meerpohl J, Norris S, Guyatt GH: GRADE guidelines. 3. Rating the quality of evidence. J Clin Epidemiol 2011;64:401-406.

Bijella MFTB, Bijella VT, Silva MSMB, Lopes ES: Avaliação clínica da aplicação de diaminofluoreto de prata a $12 \%$ (Bioride) na dentição decídua e seu efeito na incidência de cárie em primeiros molares permanentes. Rev Paul Odontol 1991;13:28-35.

Preventing Caries in Primary Teeth with SDF
Blackburn J, Morrisey MA, Sen B: Outcomes associated with early preventive dental care among Medicaid-enrolled children in Alabama. JAMA Pediatr 2017;171:335-341.

- Borenstein M, Hedges L, Higgins J, Rothstein HR: Introduction to Meta-Analysis. New York, Wiley \& Sons 2009.

Chesters RK, Ellwood RP, Biesbrock AR, Smith SR: Potential modern alternative designs for caries clinical trials (CCTs) and how these can be validated against the conventional model. J Dent Res 2004;83(spec No C):C122-C124.

- Chesters RK, Pitts NB, Matuliene G, Kvedariene A, Huntington E, Bendinskaite R, Balciuniene I, Matheson JR, Nicholson JA, Gendvilyte A, Sabalaite R, Ramanauskiene J, Savage D, Mileriene J: An abbreviated caries clinical trial design validated over 24 months. J Dent Res 2002;81:637-640.
-Chibinski AC, Wambier LM, Feltrin J, Loguercio $\mathrm{AD}$, Wambier DS, Reis A: Silver diamine fluoride has efficacy in controlling caries progression in primary teeth: a systematic review and meta-analysis. Caries Res 2017;51:527541.

Chu CH, Lo EC, Lin HC: Effectiveness of silver diamine fluoride and sodium fluoride varnish in arresting dentin caries in Chinese preschool children. J Dent Res 2002;81:767-770.

-Crystal Y, Niederman R: Silver diamine fluoride treatment considerations in children's caries management. Pediatr Dent 2016;38:466-471.

Crystal YO, Janal MN, Hamilton DS, Niederman R: Parental perceptions and acceptance of silver diamine fluoride staining. J Am Dent Assoc 2017;148:510-518.e514. 
Cury JA, Oliveira B, Santos A, Tenuta LMA: Are fluoride releasing dental materials clinically effective on caries control? Dent Mater 2015; 32:323-333.

Dos Santos VE Jr: Técnicas atraumáticas para prevenir e inativar cárie em crianças: ensaio clínico controlado; thesis, Faculdade de Odontologia de Pernambuco, Fundação Universidade de Pernambuco, Camaragibe, 2011, p 115.

Dos Santos VE Jr, de Vasconcelos FM, Ribeiro AG, Rosenblatt A: Paradigm shift in the effective treatment of caries in schoolchildren at risk. Int Dent J 2012;62:47-51.

Duangthip D, Ming J, Chu CH, Lo ECM: Nonsurgical treatment of dentin caries in preschool children - systematic review. BMC Oral Health 2015;15:44.

- Ericson D, Kidd E, McComb D, Mjor I, Noack MJ: Minimally invasive dentistry - concepts and techniques in cariology. Oral Health Prev Dent 2003;1:59-72.

Fung M, Duangthip D, Wong M, Lo E, Chu C: Arresting dentine caries with different concentration and periodicity of silver diamine fluoride. JDR Clin Trans Res 2016;1:143-148.

Gao S, Zhao I, Hiraishi N, Duangthip D, Mei M, Lo E, Chi CH: Clinical trials of silver fluoride in arresting caries among children: a systematic review. JDR Clin Trans Res 2016a;1:201210.

Gao SS, Zhang S, Mei ML, Lo EC, Chu CH: Caries remineralisation and arresting effect in children by professionally applied fluoride treatment - a systematic review. BMC Oral Health 2016b; $16: 12$.

Higgins J, Green S: Cochrane Handbook for Systematic Reviews of Interventions, ed version 5.1.0 (updated March 2011). London, Wiley \& Sons 2011.
Horst JA, Ellenikiotis H, Milgrom PL: UCSF protocol for caries arrest using silver diamine fluoride: rationale, indications and consent. J Calif Dent Assoc 2016;44:16-28.

Liberati A, Altman DG, Tetzlaff J, Mulrow C, Gotzsche PC, Ioannidis JP, Clarke M, Devereaux PJ, Kleijnen J, Moher D: The PRISMA statement for reporting systematic reviews and meta-analyses of studies that evaluate health care interventions: explanation and elaboration. PLoS Med 2009;6:e1000100.

Llodra JC, Rodriguez A, Ferrer B, Menardia V, Ramos T, Morato M: Efficacy of silver diamine fluoride for caries reduction in primary teeth and first permanent molars of schoolchildren: 36-month clinical trial. J Dent Res 2005;84:721-724.

Lo EC, Chu CH, Lin HC: A community-based caries control program for pre-school children using topical fluorides: 18-month results. J Dent Res 2001;80:2071-2074.

-Marcenes W, Kassebaum NJ, Bernabe E, Flaxman A, Naghavi M, Lopez A, Murray CJ: Global burden of oral conditions in 1990-2010: a systematic analysis. J Dent Res 2013;92:592-597.

Marinho VC, Worthington HV, Walsh T, Clarkson JE: Fluoride varnishes for preventing dental caries in children and adolescents. Cochrane Database Syst Rev 2013;7:CD002279.

Mauro S, García Robles E, Cinque C, Squassi AF, Bordoni NE: Eficiencia de tres fluoruros concentrados para la estabilización de caries de esmalte. Bol Asoc Argent Odontol Ninos 2004;33:4-11.

Mei ML, Chin-Man Lo E, Chu CH: Clinical use of silver diamine fluoride in dental treatment. Compend Contin Educ Dent 2016;37:93-98.
Mei ML, Chu CH, Lo EC, Samaranayake LP: Fluoride and silver concentrations of silver diammine fluoride solutions for dental use. Int I Paediatr Dent 2013;23:279-285.

Meyer BD, Lee JY, Lampiris LN, Mihas P, Vossers S, Divaris K: "They told me to take him somewhere else:” caregivers' experiences seeking emergency dental care for their children. Pediatr Dent 2017;39:209-214.

-Nelson T, Scott JM, Crystal YO, Berg JH, Milgrom $P$ : Silver diamine fluoride in pediatric dentistry training programs: survey of graduate program directors. Pediatr Dent 2016;38:212217

Niederman R, Huang SS, Trescher AL, Listl S: Getting the incentives right: improving oral health equity with universal school-based caries prevention. Am J Public Health 2017; 107:S50-S55.

- Nishino M, Yoshida S, Sobue S, Kato J, Nishida M: Effect of topically applied ammoniacal silver fluoride on dental caries in children. J Osaka Univ Dent Sch 1969;9:149-155.

-Schulz KF, Altman DG, Moher D, Group C: CONSORT 2010 statement: updated guidelines for reporting parallel group randomised trials. J Clin Epidemiol 2010;63:834-840.

Tedesco TK, Bonifacio CC, Calvo AF, Gimenez T, Braga MM, Raggio DP: Caries lesion prevention and arrestment in approximal surfaces in contact with glass ionomer cement restorations - a systematic review and meta-analysis. Int J Paediatr Dent 2016;26:161-172.

Zhao IS, Gao SS, Hiraishi N, Burrow MF, Duangthip D, Mei ML, Lo EC, Chu $\mathrm{CH}$ : Mechanisms of silver diamine fluoride on arresting caries: a literature review. Int Dent J 2018;68:67-76. 